\title{
Dados abertos governamentais: iniciativas e desafios na abertura de dados no Brasil e outras esferas internacionais
}

\section{Government open data: initiatives and challenges in data opening in Brazil and other international spheres}

\author{
Dirceu Flávio Macedo ${ }^{1}$, Daniela Lucas da Silva Lemos ${ }^{2}$ \\ ${ }^{1}$ Universidade Federal do Espírito Santo (UFES), Vitória, ES, Brasil. ORCID: https://orcid.org/0000-0003-4837-3618 \\ 2 Universidade Federal do Espírito Santo (UFES), Vitória, ES, Brasil. ORCID: https://orcid.org/0000-0003-1565-7366
}

Autor para correspondência/Mail to: Dirceu Flávio Macedo, dfmacedo@gmail.com

Recebido/Submitted: 06 de novembro de 2020; Aceito/Approved: 25 de janeiro de 2021

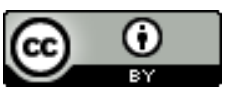

\begin{abstract}
Copyright () 2021 Macedo \& Lemos. Todo o conteúdo da Revista (incluindo-se instruções, política editorial e modelos) está sob uma licença Creative Commons Atribuição 4.0 Internacional. Ao serem publicados por esta Revista, os artigos são de livre uso em ambientes educacionais, de pesquisa e não comerciais, com atribuição de autoria obrigatória. Mais informações em http://revistas.ufpr.br/atoz/about/submissions\#copyrightNotice.
\end{abstract}

\begin{abstract}
Resumo
Introdução: A escalada na abertura de dados governamentais é um fenômeno originado a partir de extensos debates sobre a modernização do Estado, aliado à evolução tecnológica. Na literatura, constata-se uma carência de pesquisas científicas de ordem teórica e prática que apresentem cenários mais atualizados a respeito de iniciativas de abertura de dados governamentais, principalmente em ações de organização da informação nesse segmento, tanto no Brasil quanto no exterior. Objetiva apresentar antecedentes e movimento histórico de abertura de dados, além do panorama atual de iniciativas em alguns países continentais, evidenciando estágios e contribuições relevantes às discussões sobre desafios e oportunidades para seus governos. Método: Constitui uma pesquisa qualitativa, de cunho exploratório e descritivo, empregando técnicas de pesquisa bibliográfica e documental para uma revisão sistemática de cenários de dados abertos governamentais tanto no Brasil quanto em outras esferas internacionais. Resultados: Destaca as contribuições teóricas e metodológicas das Ciências da Informação e da Computação nos principais desafios mapeados na pesquisa, além do declínio de nações como os EUA e Reino Unido, outrora líderes do movimento, e a ascensão de novos protagonistas, como Coréia do Sul, França, Irlanda e Espanha, também apresentando o atual cenário brasileiro. Conclusão: Evidencia que um dos principais êxitos na abertura de dados incide na forma como os governos estão lidando com a Política de Dados Abertos Governamentais, e que aspectos internos de governança estão afetando a publicação de dados na rede, conforme preceitos do movimento aberto recomendados na literatura.
\end{abstract}

Palavras-chave: Dados Abertos Governamentais; Organização da Informação; Governança; Dados Abertos Ligados.

\begin{abstract}
Introduction: The escalation in the opening of government data is a phenomenon originated from extensive debates about the modernization of the State, combined with technological evolution. In the literature, there is a lack of scientific research of a theoretical and practical nature that presents more updated scenarios regarding initiatives to open government data, mainly in actions to organize information in this segment, both in Brazil and abroad. This article aims to present the background and historical movement of data opening, in addition to the current panorama of initiatives in some continental countries, showing relevant stages and contributions to the discussions on challenges and opportunities for their governments. Method: It is qualitative, exploratory and descriptive research using bibliographic and documentary research techniques for a systematic review of government open data scenarios both in Brazil and in other international spheres. Results: The theoretical and methodological contributions of Information and Computer Sciences are highlighted in the main challenges mapped in the research, in addition to the decline of nations such as the USA and the United Kingdom, former leaders of the movement, and the rise of new protagonists, such as Korea South, France, Ireland and Spain, also presenting the current Brazilian scenario. Conclusion: The article shows that one of the main successes in data opening concerns the way governments are dealing with Government Open Data Policy and those internal aspects of governance are affecting the publication of data on the network, according to the precepts of the open movement recommended in the literature.
\end{abstract}

Keywords: Open Government Data; Information Organization; Governance; Linked Open Data.

\section{INTRODUÇÃO}

Nos últimos anos, diversas esferas governamentais comprometidas com a modernização do Estado e os anseios da sociedade passaram a promover a abertura de seus dados apoiando-se na moderna infraestrutura da rede mundial de computadores (Gray, 2014, T. Davies, 2010).

O processo embrionário desse movimento de abertura teve seu epicentro no final do século XX e início do século XXI, quando indivíduos e organizações passam a cobrar uma postura mais aberta dos governos, exigindo maior acesso às informações. Em consonância, vislumbram progressos na defesa de causas sociais e cívicas, na produção de novas tecnologias, no fomento de inovação, no avanço científico, nos interesses econômicos, nos estudos sobre interoperabilidade, e na criação de novos formatos para acesso a dados.

O advento das Tecnologias de Informação e Comunicação (TICs) e a popularização da Internet permitiram o surgimento de um ambiente propício para questionamento e reflexão do papel do Estado perante seus cidadãos. Nesse contexto, uma nova abordagem foi requerida aos governos no esforço de estreitar sua relação com a comunidade, tornando-se inevitável seu aperfeiçoamento para um modelo mais eficiente. Na sucessão de processos 
que culminaram em um governo cada vez mais eletrônico, aflorou-se o movimento para a abertura de dados governamentais (Gray, 2014, T. G. Davies e Bawa, 2012, T. Davies, 2010).

Projetos de abertura de dados vêm sendo objeto de análise por diversas partes interessadas, incluindo organizações internacionais, ativistas, governos e pesquisadores de variadas áreas do conhecimento na busca de se compreender os preceitos estabelecidos pelo movimento aberto; se estão de fato trazendo benefícios e transformações sociais pleiteadas; ou se estão atendendo as melhores práticas de publicação de dados na rede, como o padrão Linked Open Data, que prioriza a ligação entre bases de dados de fontes heterogêneas (Machado, Souza, e Graça Simões, 2019, Bizer, Health, e Berners-Lee, 2009).

A abertura de dados pode ser encarada como um grande movimento para compartilhamento de conhecimento e ampliação democrática, beneficiando tanto a sociedade quanto o governo no aspecto de acessibilidade a fontes de dados de interesse (Bauer \& Kaltenböck, 2011), o que aumenta a responsabilidade, a transparência, a eficiência governamental, o empoderamento cívico, além de incentivar o uso e a reutilização de informações para ações de interesse (Attard, Orlandi, Scerri, e Auer, 2015, Open Government Partnership, 2011).

Entretanto, são muitos os desafios que os governos vêm lidando para abrir seus dados (Pinto e Almeida, 2020; Kučera, Chlapek, Klímek, e Necaský, 2015, Santarem Segundo, 2015), tanto do ponto de vista técnico associado à implantação, aos princípios e aos formatos, quanto em relação às estratégias de governança digital, exigindo-se um grande esforço interdisciplinar para a disponibilização dos dados governamentais na rede sem impor riscos associados à integridade e à confiabilidade dos dados (Shepherd et al., 2019). Parte-se da hipótese nesta pesquisa de que dados governamentais são publicados na rede por grande parte das nações de forma precária, tanto em aspectos tecnológicos e licenças de uso para acesso e reúso de dados quanto nas práticas de organização da informação estabelecidas.

Pesquisas têm sido desenvolvidas progressivamente nos campos das Ciências da Informação (CI) e da Computação (CC), visando a estudos sobre a problemática do excesso de informações e sua organização, com o objetivo de aumentar o escopo da interoperabilidade e da integração plena de informações heterogêneas entre sistemas e bases de dados disponíveis na Web (D. L. Lemos \& Souza, 2020). A demanda por integração e compartilhamento de dados heterogêneos na rede tem despertado interesse em instituições de governo, que buscam tornar mais efetivo o acesso e a recuperação de informações por meio de tecnologias mais sofisticadas (Pinto \& Almeida, 2020).

Em análise prévia da literatura nos dois campos supracitados, constatou-se uma carência de pesquisas científicas de ordem teórica e prática que apresentem cenários mais atualizados a respeito de iniciativas de abertura de dados governamentais que evidenciem, principalmente, ações de organização da informação nesse segmento, tanto no Brasil quanto no exterior. Assim sendo, o artigo buscou compreender, por meio de revisão de literatura, a confluência de publicações de dados abertos governamentais relacionadas às iniciativas e desafios tanto no Brasil quanto em outras esferas internacionais, o que levou a formular a seguinte questão de pesquisa: como as instituições de governo em âmbito mundial estão conduzindo suas práticas de publicações de dados abertos, levando-se em consideração todo um legado histórico para o que se pratica atualmente?

Nesse viés, o objetivo do artigo é apresentar antecedentes e uma abordagem histórica que subjaz o movimento de dados abertos em vários países levando-se a um panorama atual de esforços locais, evidenciando estágios e contribuições relevantes às discussões sobre desafios e oportunidades para os governos na busca de abertura de seus dados à sociedade contemporânea.

A principal contribuição desta pesquisa é a sistematização de um conjunto de desafios e oportunidades mapeados nas ações de abertura de dados governamentais em alguns países a fim de estreitar a relação entre a esfera pública e a sociedade. Considera-se que a publicização de dados abertos é de extrema importância para a ampliação democrática na sociedade, contudo a literatura no campo de dados abertos governamentais carece de estudos do estado da arte mais reflexivos e alinhados a contribuições interdisciplinares oriundas especialmente da CI e da CC.

\section{ORGANIZAÇÃO DE DADOS ABERTOS EM REDE - CONTRIBUIÇÕES DA CI E DA CC}

Organização e representação da informação e do conhecimento em contextos digitais é uma área que vem sido desenvolvida de maneira interdisciplinar pela CI e CC cujos esforços se concentram em melhorias aos sistemas de recuperação da informação na Web, objetivando conteúdos semânticos e interoperáveis neste ambiente.

Em CI, a ênfase recai sobre princípios teóricos e metodológicos voltados à organização da informação por meio de suas representações descritivas (Gilliland, 2016, Joudrey, Taylor, e Miller, 2015) e temática (González, 2011, Lancaster, 1986). Ambas geralmente são vinculadas a processos de catalogação de documentos digitais que resultam na produção e no uso de metadados (e seus padrões) e linguagens documentárias fundamentados em normas e modelos conceituais seminais ao campo. O propósito desse tipo de tratamento da informação é retratar aspectos específicos do documento que permitam a sua individualização e determinação de pontos de acesso a 
fim de proporcionar aos usuários a condição de encontrar, identificar, selecionar e obter o item (International Federation of Library Associations and Institutions, 2009).

Já na CC, a Web Semântica, suas tecnologias subjacentes e a proposta de dados abertos interligados (Machado et al., 2019, Bizer et al., \& Berners-Lee, 2009; Berners-Lee, Hendler, e Lassila, 2001) são esforços de pesquisas para propor soluções tecnológicas eficientes para a organização semântica de recursos em mídias diversas, destacando as ontologias como sistemas de organização do conhecimento em potencial (D. L. Lemos \& Souza, 2020), que viabilizam o intercâmbio semântico de diferentes bases de dados e sistemas de informação presentes na rede.

No cerne dos conceitos dessa Web semanticamente organizada está a interoperabilidade, que pode ser compreendida como a capacidade de diversos sistemas de informação e organizações trabalharem em conjunto de modo a potencializar que pessoas, organizações e sistemas computacionais interajam para trocar informações de maneira eficaz e eficiente (D. L. S. Lemos, Mendonça, \& Souza, 2020). Tratar a interoperabilidade é uma das principais metas atuais do World Wide Web Consortium (W3C), que vem se dedicando ao desenvolvimento de metodologias, padrões de metadados e tecnologias semânticas para avanços na Web atual, melhorando o significado dos conteúdos na rede (Machado et al., 2019).

Nessa perspectiva, destaca-se o modelo ou padrão Linked Open Data (LOD) (Bizer et al., 2009) que propõe um conjunto de princípios e tecnologias visando apoiar a publicação de dados abertos na Web. O LOD opera com padrões internacionais (subjacentes à Web Semântica), melhores práticas de publicação, disseminação e reutilização de dados estruturados, fornecendo um mecanismo simples para combinar dados de várias fontes heterogêneas na Web visando acesso e consumo dessas fontes tanto por humanos quanto por máquinas. Para tal, Tim Berners-Lee, seu idealizador, recomenda um sistema de ranking ou modelo cinco estrelas ${ }^{1}$, que ajuda a diagnosticar o nível de abertura de dados na rede, fornecendo degraus alcançáveis para se chegar a níveis mais refinados de dados abertos. Rautenberg, Souza, Dall'Agnol, e Michelon (2019) destacam esses níveis:

- Uma estrela: dados publicados sob licença aberta, não importando o formato de publicação (ex.: consumo de dados disponibilizados em um arquivo no formato PDF).

- Duas estrelas: recursos disponíveis como dados estruturados em formatos legíveis por máquina (ex.: formato de uma planilha XLS).

- Três estrelas: dados publicados em formato aberto não proprietário (ex.: arquivo CSV em vez de XLS).

- Quatro estrelas: dados nomeados com Uniform Resource Identifier - URI para identificação de recursos na rede. Permite que outros usuários criem links entre os dados, possibilitando reúso de parte ou de todos os dados (ex.: reutilização de vocabulários padronizados para descrição de dados em formato Resource Description Framework - RDF).

- Cinco estrelas: dados interligados a outros dados de fontes externas com semântica bem definida para prover contexto (ex.: uso e reúso de ontologias de domínios específicos para organização e interligação semântica de conteúdos dos datasets)

A prática de LOD no âmbito governamental adquire uma nova dimensão, em especial pela grande capacidade dos governos em centralizar e coletar uma vasta gama de dados e informações sensíveis a toda uma sociedade. Nas últimas décadas, organizações, governos, pesquisadores e especialistas dedicaram-se no aprofundamento do tema "dados abertos governamentais" e na consolidação de suas definições (Pinto \& Almeida, 2020).

Berners-Lee (2009) corrobora ponderando que dados governamentais devem ser ofertados na Web por três motivos: (i) aumenta a conscientização dos cidadãos sobre as funções do governo, permitindo maior responsabilização; (ii) contribui com informações valiosas sobre o mundo; e (iii) permite que os governos e o mundo funcionem com mais eficiência.

\section{METODOLOGIA DE PESQUISA}

A presente pesquisa pode ser classificada segundo seu problema, seus objetivos e seus procedimentos técnicos para coleta e análise dos dados, de forma a confrontar a visão teórica com os dados da realidade (Gil, 2019).

Com base na abordagem do problema, esta pesquisa pode ser classificada como sendo de natureza qualitativa em vista à necessidade de analisar, interpretar e descrever um cenário em sua realidade corrente. Com relação aos seus objetivos, a pesquisa pode ser classificada em duas tipologias, a saber: exploratória e descritiva. Na primeira, buscou-se averiguar de que forma os governos estão publicando seus estoques informacionais na rede, mapeando oportunidades e desafios; na segunda, buscou-se descrever o fenômeno investigado em recortes temporais. E em relação aos procedimentos técnicos para a coleta e análise de dados, utilizou-se de pesquisa bibliográfica para fundamentar conceitos, fornecer sustentabilidade teórica ao estudo e viabilizar a proposição de uma revisão de literatura sobre cenários de dados abertos governamentais a nível Brasil e internacional. Utilizou-se também

\footnotetext{
${ }^{1}$ https://5stardata.info/pt-BR/
} 
de pesquisa documental para a seleção de material institucional oriundo de algum projeto analisado, incluindo datasets, manuais e relatórios de pesquisas.

Dada a natureza interdisciplinar da pesquisa, delimitou-se o recorte temático nos campos da CI e CC por possuírem um aporte teórico metodológico consolidado e relevante às práticas de abertura de dados, especialmente na área de organização e representação da informação e do conhecimento em ambientes digitais. O campo da Administração Pública também foi investigado como complemento aos da informação e da tecnologia no aspecto de governança e política de dados. Procedeu-se, então, a um levantamento nesses campos em bases de dados representativas consultadas por meio do Portal de Periódico da Capes, a saber: SciELO; Library and Information Science Abstracts (LISA); Base de Dados em Ciência da Informação (BRAPCI); Web of Science; Computer and Information Systems; Computers and Applied Sciences Complete; Information Science \& Technology Abstracts (ISTA); Library, Information Science \& Technology Abstracts with Full Text; e Scopus (Elsevier B.V).

Em adição, foram consultadas fontes de informação relevantes, tais como: os Anais do Encontro Nacional de Pesquisa em Ciência da Informação (Enancib), visto como um dos principais eventos científicos nesse campo no país; repositórios de governos nacionais e de organismos internacionais envolvidos com a abertura de dados governamentais (World Wide Web Consortium, Open Government Partnership, European Union, dentre outros).

Como critérios de busca nas bases de dados, as principais palavras-chave utilizadas em inglês foram "Open Government Data" conjuntamente com seu análogo em português "Dados Abertos Governamentais", ainda adicionando os termos "OGD", "state of the art", "systematic", "barriers" e "challenges". O critério para a data de publicação foi dividido em um período de dez anos para trabalhos relacionados ao movimento histórico para abertura de dados governamentais, e de cinco anos para o recorte do panorama atual a fim de evidenciar o que se fez no campo nos últimos anos.

Cerca de 100 publicações entraram nos critérios, sendo selecionadas 30 em função de análise prévia do resumo e palavras-chave. As publicações descartadas encontravam-se ou repetidas ou com temáticas não relacionadas de forma explícita com a proposta do artigo em levantar as iniciativas e desafios do movimento de dados abertos governamentais.

A etapa de coleta e análise dos dados contou com o método de pesquisa conhecido como análise de conteúdo (Bardin, 2016), que adota como estratégia um conjunto de métodos e técnicas com abordagens quali-quantitativas, visando à criação de categorias de análise para compreensão mais abrangente do fenômeno investigado. Desse modo, as categorias analíticas que orientaram na extração, análise e interpretação dos dados presentes nos materiais selecionados no estudo foram as seguintes: antecedente, local, cronologia, iniciativa e desafio.

\section{DADOS ABERTOS GOVERNAMENTAIS}

\section{Antecedentes e Aspectos Históricos}

O tema "dados abertos" surgiu pela primeira vez em 1995 a partir de um documento pertencente a uma agência científica dos Estados Unidos (EUA) que promovia troca aberta de dados geofísicos e ambientais entre agências governamentais para melhor compreensão de fenômenos associados. A abertura de dados pode ser considerada uma práxis da comunidade científica que já era defendida pelo pesquisador Robert King Merton ${ }^{2}$, o qual destacava a ampliação do conhecimento humano como um grande benefício à sociedade. Desse modo, os pesquisadores foram os primeiros a perceber os benefícios da abertura e compartilhamento de dados, contudo foi o encontro entre teorias científicas e conceitos de software livre e código aberto que moldaram o panorama de dados abertos a que conhecemos hoje (Chignard, 2013).

Gray (2014, p. 5-6) apresenta algumas evidências que contribuíram para o paradigma de abertura de dados nos anos de 1990 e início dos anos 2000, sobretudo na Europa e nos EUA, a saber: i) defesa de políticas e debates promovidos por ativistas, tecnólogos e hackers cívicos ${ }^{3}$ a respeito da infraestrutura governamental e do fornecimento de informações públicas; ii) discussões sobre políticas de informação do setor público; iii) debates sobre o potencial econômico e social das informações e dados geoespaciais, por meio dos quais viabilizaram discussões acerca de seus padrões, licenças e formatos. Em paralelo, a evolução das TICs colocou a tecnologia digital e a Internet como protagonistas da digitalização de processos, impactando os governos no que diz respeito à democratização de acesso e à transparência em dados governamentais na rede (Jetzek, Avital, \& Bjørn-Andersen, 2014).

Os governos se valeram da evolução das TICs, especialmente do potencial da Internet, na democratização do acesso à informação pela sociedade, cujo fenômeno é denominado governo eletrônico (e-Gov) e, atualmente, conceituado como governo digital (Pinto \& Almeida, 2020). A infraestrutura oferecida pelo e-Gov acaba por impulsionar a abertura de dados governamentais, sendo ofertada como mais um canal essencial para o público com foco na democratização e transparência das atividades governamentais. No caso brasileiro, os canais de

\footnotetext{
${ }^{2}$ Robert King Merton é considerado um dos pais da sociologia da ciência.

${ }^{3}$ Indivíduos que exercem um ativismo cívico empregando avançados conhecimentos computacionais.
} 
transparência e dados abertos caminham intimamente relacionados com a política de e-Gov do país (Pinto \& Almeida, 2020).

O movimento para abertura de dados governamentais deu um grande passo em 2007 quando em Sebastopol, Califórnia, EUA, ocorreu uma reunião de "advogados do governo aberto" objetivando criar uma definição para dados abertos, e assim contribuir para orientar os governos em suas iniciativas de abertura (Gray, 2014). A partir daí, o conceito de "dados abertos" migrou de uma investigação de ativistas e técnicos para o discurso de autoridades governamentais.

Desde então, pautas referentes à abertura de dados governamentais passaram a compor a agenda de grandes organizações internacionais, como o G8 (Grupo dos países mais industrializados do mundo), o G20 (Grupo das maiores economias do mundo), a OCDE (Organização para a Cooperação e Desenvolvimento Econômico) e o Banco Mundial (Gray, 2014).

Em 2009, deu-se a publicação do "Memorando sobre Transparência e Governo Aberto" pelo então presidente dos EUA, Barack Obama (Schrock, 2016). O memorando colocou a cultura aberta no centro da ação pública, pleiteando seus princípios fundamentais, como transparência, participação e colaboração (Chignard, 2013), além de estabelecer uma cooperação moderna entre políticos, administração pública, indústria e cidadãos.

Em 2011, o Open Government Partnership (OGP) foi lançado quando um grupo de oito países fundadores (Brasil, Indonésia, México, Noruega, Filipinas, África do Sul, Reino Unido e EUA) endossou a Declaração do Governo Aberto (Bauer \& Kaltenböck, 2011). O OGP - que em julho de 2020 contabilizava 78 países - reconhece que as pessoas estão exigindo maior participação cívica e acesso às informações governamentais.

Em 2013, o compromisso de tornar os dados abertos foi reafirmado na cúpula do G8 por meio da "Carta Aberta de Dados" $"$, estabelecendo alguns princípios, dentre os quais de que os dados sejam abertos por meio de padrões, entendendo que existem legislações sobre propriedade intelectual e privacidade que devem ser observadas. Nesse mesmo ano, os EUA tornaram aberto e legível por máquina o novo padrão de informações do governo.

No que tange ao Brasil, as iniciativas de abertura de dados governamentais surgiram de forma discreta a partir de 2005 (Silva \& Pinheiro, 2015). Desde então, o governo passou a intencionar maior transparência por meio da publicação de informações de interesse da sociedade na Web (Victorino et al., 2017). Por exemplo, em 2006 a Controladoria Geral da União (CGU) determinou que os órgãos e entidades da Administração Pública Federal mantivessem em seus portais, informações atualizadas e detalhadas sobre determinados temas, tais como execução orçamentária, licitações, contratações, entre outros.

Em 2009, tem-se o primeiro marco no movimento de abertura no Brasil através do Decreto Federal $n^{\circ} 6.932$, determinando, ao Poder Executivo, a disponibilização de suas informações e bases de dados aos órgãos e entidades públicas, além de a aplicação de soluções tecnológicas para viabilizar tal compartilhamento (Ribeiro \& Almeida, 2011).

No entanto, somente após ser sancionada a Lei 12.527/2011 (Lei de Acesso à Informação Pública - LAI) em 2011 é que se iniciou a disponibilização de dados governamentais abertos, regularizando o acesso a esse tipo de conteúdo ao público em geral (Silva \& Pinheiro, 2015). Ainda em 2011, o governo brasileiro se comprometeu com a iniciativa OGP, lançando em seguida seu Portal Brasileiro de Dados Abertos (2020). As iniciativas de abertura de dados pelo governo brasileiro repercutiram pela América Latina, influenciando o México e o Chile em projetos similares (Young \& Verhulst, 2016). Em 2016, o Tribunal de Contas da União (TCU), visando aumentar a conscientização sobre a cultura de dados, elaborou uma publicação com cinco motivos para abertura de dados na Administração Pública: i) exigência de maior transparência da gestão pública pela sociedade; ii) contribuição da própria sociedade com serviços inovadores para o cidadão; iii) qualidade dos dados; iv) novos negócios; e v) obrigatoriedade legal. Também em 2016 foi instituída a Política de Dados Abertos do Poder Executivo Federal (Decreto No 8.777), criando a Infraestrutura Nacional de Dados Abertos (INDA) ${ }^{5}$ e o Plano de Dados Abertos (PDA) - um instrumento que operacionaliza a Política de Dados Abertos Governamentais (Ministério do Planejamento, 2018).

Logo, tem-se que o moderno significado de "abertura" para dados governamentais, bem como o contexto que vivenciamos atualmente é resultado de propostas engajadas de reformas, iniciativas e experiências sucessivas de abertura de dados em diversos campos de atuação ao redor do mundo. Somam-se a isso, o avanço e o uso das TICs por meio das quais se aprimorou a qualidade dos sistemas de informação do governo e, consequentemente, ampliou-se o debate e a participação do cidadão na construção de políticas públicas (Pinto \& Almeida, 2020, p. 5). Em decorrência desse legado, o panorama atual associado a Dados Abertos Governamentais em contexto mundial, incluindo o Brasil, é elucidado na próxima seção.

\footnotetext{
${ }^{4}$ https://www.gov.uk/government/publications/open-data-charter

${ }^{5}$ Conjunto de padrões, tecnologias, procedimentos e mecanismos de controle necessários para atender às condições de disseminação e compartilhamento de dados e informações públicas no modelo de Dados Abertos. https://www.gov.br/governodigital/pt-br/dadosabertos/infraestrutura-nacional-de-dados-abertos
} 


\section{PANORAMA ATUAL: DADOS ABERTOS GOVERNAMENTAIS EM ÂMBITO MUNDIAL}

Essa seção busca descrever o cenário atual em países com papéis legados e preponderantes ao progresso de Dados Abertos Governamentais em escala mundial, como EUA e Reino Unido, além de países que se destacam em seus respectivos continentes, como são os casos do Brasil na América Latina, da Coréia do Sul na Ásia, do Quênia na África, da Austrália na Oceania, e Áustria, Alemanha, República Tcheca, Irlanda, Espanha e França na Europa.

Até outubro de 2020, a organização DataPortals.org ${ }^{6}$ já contabilizava cerca de 590 portais de dados abertos ao redor do mundo. Estudo recente de Yi (2019) relata que o portal de dados abertos dos $\mathrm{EUA}^{7}$ dispunha em 2018 de 341.925 datasets. Já sobre o portal de dados abertos do Reino Unido ${ }^{8}$, recente pesquisa conduzida por Huang, Wang, Zhang, Wu, e Xie (2019) indicou que até 2017 o sítio contava com mais de 40.000 datasets. Até agosto de 2020 já existiam cerca de 400 aplicativos desenvolvidos baseados em dados abertos do Reino Unido.

Outra tendência é a integração de projetos nacionais em uma única plataforma intergovernamental, como o European Data Portal ${ }^{9}$, o Open Data for Africa, e o Latin American Open Data Initiative (Kassen, 2018). Também surgiu a partir de iniciativas de organizações governamentais e não governamentais uma série de painéis e relatórios que se propõem a medir a maturidade e o engajamento dos governos na abertura de dados, dando destaque ao: Open Data Maturity Report 2019 (Blank, 2019); OURdata Index 2019 (OECD, 2020); Open Data Barometer $^{10}$; Open Data Monitor ${ }^{11}$; e Global Open Data Index ${ }^{12}$.

O Open Data Barometer, por exemplo, mede as iniciativas de países que se comprometeram formalmente com a abertura de dados, seguindo os Princípios da Carta Internacional de Dados Abertos ${ }^{13}$, para os quais estabelecem que os dados sejam: abertos por padrão, relevantes, publicados com celeridade, acessíveis, reutilizáveis e interoperáveis. Usando uma metodologia própria, aplicam notas que variam de 0 (zero) a 100, incluindo políticas bem definidas para dados, consistência no gerenciamento e na publicação de dados, execução das políticas públicas de dados abertos pelo país e treinamentos sobre dados abertos.

Observando alguns desses indicadores, afere-se que países como os EUA e o Reino Unido, outrora líderes em atividades e iniciativas de dados abertos, acabaram, com o passar dos anos, perdendo espaço em termos de participação. A edição de 2018 do relatório do Open Data Barometer externa preocupação, especialmente pelo fato de ambos os governos assumirem compromissos de abertura e, no entanto, estarem indo na contramão dos Princípios da Carta Internacional de Dados Abertos.

A Tabela 1 apresenta dados monitorados pelo Open Data Barometer em que se observa a queda do Reino Unido e dos EUA, a ascensão de nações como o Canadá, a França, a Austrália e a Coréia do Sul, além da colocação do Brasil.

\begin{tabular}{|c|c|c|c|}
\hline Posição & País & Pontuação & Alteração de Pontuação \\
\hline $1^{\circ}$ & Canadá & 76 & +18 \\
\hline $2^{\circ}$ & Reino Unido & 76 & -4 \\
\hline $3^{\circ}$ & Austrália & 75 & +17 \\
\hline $4^{\circ}$ & França & 72 & +17 \\
\hline $5^{\circ}$ & Coréia do Sul & 72 & +25 \\
\hline $9^{\circ}$ & Estados Unidos & 64 & -11 \\
\hline $14^{\circ}$ & Brasil & 50 & +15 \\
\hline
\end{tabular}

Tabela 1. Dados performáticos de algumas nações segundo o Open Data Barometer.

Fonte: Adaptado do Open Data Barometer. https://opendatabarometer.org/ - janeiro de 2021.

Na Tabela 2, o OURdata Index 2019 aponta que o Reino Unido (líder em 2017) não sustentou uma agenda de dados abertos na sua política pública, caindo para a $20^{\circ}$ posição. Os EUA, à época, não estavam disponíveis nessa pesquisa.

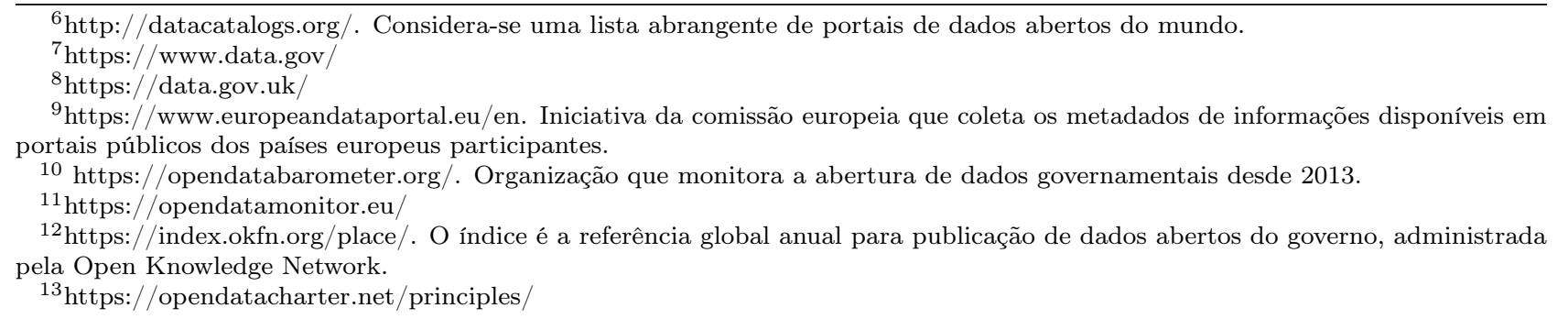




\begin{tabular}{|c|c|c|c|}
\hline Posição & País & Pontuação & Alteração de Pontuação \\
\hline $1^{\circ}$ & Coréia do Sul & 0.93 & -0.01 \\
\hline $2^{\circ}$ & França & 0.90 & +0.05 \\
\hline $3^{\circ}$ & Irlanda & 0.77 & +0.24 \\
\hline $4^{\circ}$ & Japão & 0.75 & -0.05 \\
\hline $5^{\circ}$ & Canadá & 0.73 & +0.03 \\
\hline $2^{\circ}$ & Reino Unido & 0.57 & -0.22 \\
\hline
\end{tabular}

Tabela 2. Queda acentuada do Reino Unido no índice OURdata Index. Fonte: Adaptado do OURdata Index 2019.

Em que pese a posição intermediária do Brasil no Open Data Barometer, é louvável o seu crescimento nos últimos anos, tendo ainda uma grande margem a ser explorada no aperfeiçoamento da qualidade de seus dados abertos. Com relação ao índice OURdata Index, o país estava indisponível por não ser membro da Organisation for Economic Co-operation and Development (OECD).

Os estudos acadêmicos corroboram os monitores. Ao analisarem 400 datasets do portal do Reino Unido, V. Wang e Shepherd (2020) constataram sérios problemas na oferta de dados, apontando que a maioria dos recursos informacionais disponíveis nos últimos 42 meses (cerca de 70\%) é representada por dados fornecidos no nível um, segundo o ranking cinco estrelas (Bizer et al., 2009), contra apenas 30\% de dados estruturados e granulares.

Logo, conclui-se que mesmo os entusiastas do governo aberto enfrentam grandes obstáculos para publicação. Evidencia-se uma série de confusões organizacionais no Reino Unido, seja para localizar a responsabilidade da Política de Dados Abertos do Governo, seja sobre a definição de quais profissionais devem liderar a atividade, ou sobre as abordagens para publicação (Shepherd et al., 2019). Sobre essa situação, V. Wang, Shepherd, e Button (2019, p. 60) afirmam que: "Se o Reino Unido, como pioneiro da iniciativa de dados abertos, está sobrecarregado com esses problemas, então os países menos avançados provavelmente experimentarão obstáculos iguais ou semelhantes".

A realidade americana diagnosticada por Luna-Reyes e Najafabadi (2019, p. 178) atesta que a falta de clareza da Política de Dados Abertos Governamentais "[...] levou a um período de estagnação no desenvolvimento do programa de dados abertos do governo". Soma-se a esse fato a carência de envolvimento coordenado das diversas agências para compreender as necessidades de requisitos de dados de suas respectivas comunidades. Yi (2019), por exemplo, alerta que os EUA publicaram mais de $50 \%$ dos seus dados abertos em formatos que inviabilizam a leitura por máquinas, comprometendo a sua reutilização. Em contrapartida, o vizinho Canadá destaca-se com suas taxas de abertura de dados e transparência, contando com grandes empresas - em número superior aos dos EUA - engajadas em apoiar ONGs no uso de dados abertos governamentais (Young \& Verhulst, 2016).

Na África, o movimento de abertura de dados desacelerou, estando, porém, o Quênia na liderança em aspectos técnicos, com um portal de dados com recursos similares aos do Reino Unido e dos EUA (Afful-Dadzie \& Afful-Dadzie, 2017).

De acordo com Chatfield e Reddick (2018), na Austrália, o governo central é o agente inovador para a abertura de dados frente aos sete governos estaduais, realizando investimentos em infraestrutura tecnológica como a plataforma CKAN (Comprehensive Knowledge Archive Network). Em complemento, as iniciativas estaduais pioneiras tiveram em comum a ação de gestores públicos na difusão da nova cultura de abertura entre os diferentes setores.

Partindo para o continente europeu, a Áustria se destaca na criação de um Modelo de Implementação de Governo Aberto que serve de base para projetos locais quanto para outras nações, sendo reaproveitado para organizar processos de publicação de dados (Blank, 2019). Seu portal de dados abertos prima tanto pela qualidade, semântica, relevância dos dados (Saez Martin, Rosario, \& Pérez, 2016) quanto pelo seu aspecto técnico, com ótimos indicadores de segurança e eficiência na infraestrutura de TI (Juana-Espinosa \& Luján-Mora, 2019).

Na Alemanha, a Política Nacional de Dados Abertos em nível federal vigora desde 2017, contemplando a abordagem aberta por padrão para dados coletados por agências federais (Blank, 2019, OECD, 2019). Kassen (2018), no entanto, alerta que o governo central outorga autonomia aos demais governos locais no desenvolvimento de iniciativas próprias, o que resulta na profusão de Políticas de Dados Abertos no país.

As iniciativas de abertura na Alemanha possuem barreiras, como a falta de padronização da interface gráfica e dos formatos de dados entre os inúmeros portais governamentais existentes no país, afetando a usabilidade (Vetrò et al., 2016). Wirtz, Piehler, Thomas, e Daiser (2016) também apontam severas barreiras organizacionais (legais, burocráticas, hierárquicas, sentimento de empregos ameaçados) que estão minando a atuação dos servidores públicos no esforço de cooperação para iniciativas de dados governamentais abertos no país.

Já na República Tcheca, o Catálogo Nacional de Dados Abertos ${ }^{14}$ criado em 2015 tem a incumbência de reunir

\footnotetext{
${ }^{14}$ https://data.gov.cz/
} 
os datasets governamentais, sanando dessa forma a grande descentralização das fontes de dados do país (Pavlík, Hrnčírová, Stočes, Masner, \& Vaněk, 2020). O modelo de governança adotado para a publicação de dados abertos em todos os níveis de governo iniciou com forte coordenação central (de cima para baixo), mas atualmente os governos locais conduzem suas próprias iniciativas de dados abertos (Blank, 2019).

O portal Tcheco também dispõe de uma área para exibir a qualidade dos metadados usados, além de possuir 100\% de adesão ao padrão DCAT-P (Data Catalog Vocabulary Application Profile), conformidade esta que melhora o uso, a descoberta e a reutilização de dados abertos em portais da Europa (Blank, 2019).

Não obstante, Blank (2019) expõe uma lacuna na iniciativa Tcheca relacionada ao fato de o portal só publicar dados de 30 entes públicos, dentro de um universo de cerca de 10.000. Isso ocorreria pelo fato de a maioria dos pequenos órgãos não ter a capacidade de publicar seus dados. Pavlík et al. (2020) complementam a crítica a determinados pontos do portal federal, a saber: alguns formatos de dados disponíveis comprometem a interoperabilidade; muitos datasets encobertos por outros; além de que o acesso a alguns tipos de dados brutos só estão disponíveis para o público mediante pagamento de taxa.

Mais recentemente, países como Irlanda, Espanha e França também despontaram, possuindo desde 2018 uma liderança no continente, sendo únicas na criação de tendências (Blank, 2019). Ao contrário da Alemanha e República Tcheca, que restringem principalmente aos representantes do setor público a participação em seus modelos de governança, a Irlanda e a Espanha, de maneira inovadora, contemplam em seus modelos a participação de atores tanto do setor privado, quanto do terceiro setor (Blank, 2019). No aspecto interativo, os portais nacionais da França, Áustria e República Tcheca permitem que os usuários publiquem seus datasets no sítio, transformando-os em verdadeiras plataformas colaborativas (OCDE, 2019).

Em se tratando de iniciativas em LOD, a Europa ainda não se envolveu conforme os preceitos recomendados (Blank, 2019). Segundo o Relatório de Maturidade de Dados Abertos do European Union "A porcentagem de dados abertos ligados é inferior a 10\% em 27 países (96\%). Somente na Letônia, entre $10 \%$ a $19 \%$ dos dados têm cinco estrelas" (p. 58, tradução nossa). Em se tratando do nível de quatro estrelas, a Alemanha desponta na Europa nesse quesito, com cerca de $60 \%$ a $70 \%$ dos dados disponibilizados nessa especificação.

No espectro de licenças de dados, as abordagens na União Europeia são distintas, com grande parte dos membros (75\%) recomendando aos seus editores o uso de licenças Creative Commons (CC). Já a França, a Alemanha, a Romênia, a Espanha e o Reino Unido recomendam a licença aberta desenvolvida pelo próprio país, sendo que a justificativa francesa é que o licenciamento CC restringe a reutilização de dados (Blank, 2019).

Apesar de grande parte da literatura sobre Dados Abertos Governamentais indicar países ocidentais (Altayar, 2018), é possível também identificar iniciativas emergentes em países do Oriente Médio e Ásia, conforme relatos a seguir.

No continente Asiático desponta a Coréia do Sul, tido como um país exemplo, de alta capacidade, experiência e maturidade (Altayar, 2018). Em 2018, o portal de dados abertos da Coréia do Sul ${ }^{15}$ possuía 18.528 datasets, com cerca de 51\% deles legíveis por máquina e não proprietários (Yi, 2019). A China acenou com avanços a partir de 2016 com uma política de big data e dados abertos governamentais. No entanto, o país ainda não dispõe de um portal nacional unificado, contando com 19 sítios locais ainda em seu início - com Taiwan e Hong Kong como províncias mais avançadas que as demais (D. Wang, Chen, \& Richards, 2018).

No Oriente Médio, os países que compõem o Conselho de Cooperação do Golfo (CCG) - Bahrain, Kuwait, Catar, Omã, Arábia Saudita e Emirados Árabes Unidos - estão em seu estágio inicial e despertam pouco interesse acadêmico (Saxena, 2017). A Arábia Saudita, por exemplo, é uma iniciativa unilateral do lado da oferta (governo), ou seja, desconsidera-se qualquer outro tipo de necessidade ou clamor que porventura possa ser do interesse da sociedade e demais partes interessadas (Altayar, 2018).

Já no Brasil, o maior expoente na disseminação e publicação de dados abertos no Governo Federal é o Portal Brasileiro de Dados Abertos (2020), que até outubro de 2020 já contabilizava cerca de 9850 conjuntos de dados, impulsionado pela plataforma CKAN.

A Política de Dados Abertos do Poder Executivo Federal tem em seu PDA um instrumento que padroniza os processos de publicação de dados abertos, com a obrigação de cada órgão/entidade elaborar um plano com vigência de dois anos. O Ministério do PlanejamentoMinistério do Planejamento, por exemplo, tem em seu PDA estratégias de abertura, governança, monitoramento e controle.

No entanto, analisando o monitor da $\mathrm{CGU}^{16}$ para controle das iniciativas de abertura do Poder Executivo Federal, constata-se que até outubro de 2020 registravam-se 141 órgãos que ainda não haviam se manifestado diante da criação de um PDA, contra 73 órgãos de acordo e oito em construção.

Partindo do painel da CGU, Silva e Pinheiro (2019) selecionaram através de critérios predefinidos, dentre eles a adesão de $100 \%$ ao Plano de Dados Abertos Governamentais, amostras de datasets oriundos de três órgãos

\footnotetext{
${ }^{15}$ https://www.data.go.kr/

${ }^{16}$ http://paineis.cgu.gov.br/dadosabertos/index.htm
} 
federais, e submetendo-os posteriormente à avaliação da DGAbr, uma métrica brasileira criada para avaliar o potencial de reúso dos dados abertos governamentais disponibilizados no Brasil. Observaram a existência de datasets incompletos, com uma série temporal inexistente, desatualizados e sem qualquer aderência aos preceitos do LOD. Contudo, consideram que a abertura de dados por parte do governo é capaz de trazer informações relevantes à sociedade, como dados sobre recursos hídricos, ou mesmo orçamento federal.

Em complemento, os resultados expostos por Macedo e Lemos (2019) sobre projetos voltados à publicização de Dados Abertos Governamentais nas esferas públicas brasileiras sugerem que a preocupação é dispor os dados na Web em formatos não proprietários e legíveis por máquina. Porém, em relação aos preceitos do LOD, existe uma baixa adesão do Governo Federal em oferecer os dados abertos em um formato compatível. A situação se agrava nas esferas Estaduais e Municipais, em que a padronização é praticamente nula.

Observando os exemplos de abertura de dados governamentais nas esferas internacionais, percebe-se que as ações mais bem-sucedidas possuem suporte não apenas de boas práticas e tecnologias avançadas, mas também de políticas públicas bem alinhadas com os interesses da abertura informacional, o que reverbera positivamente em todo o ciclo de vida dos dados. Essa visão é ratificada pela "Declaração de 360 dos Dados ${ }^{17}$ " elaborada em 2019 pelas Nações Digitais (Digital Nations), uma cúpula de países comprometidos com a modernidade governamental em que enfatiza a necessidade de uma ampla visão para o gerenciamento de dados abertos governamentais.

\section{DISCUSSÕES}

A partir dos resultados alcançados com a revisão de literatura, o progresso da abertura de dados governamentais foi organizado em uma linha cronológica (Figura 1) evidenciando marcos de abertura de dados tanto no Brasil quanto no exterior.

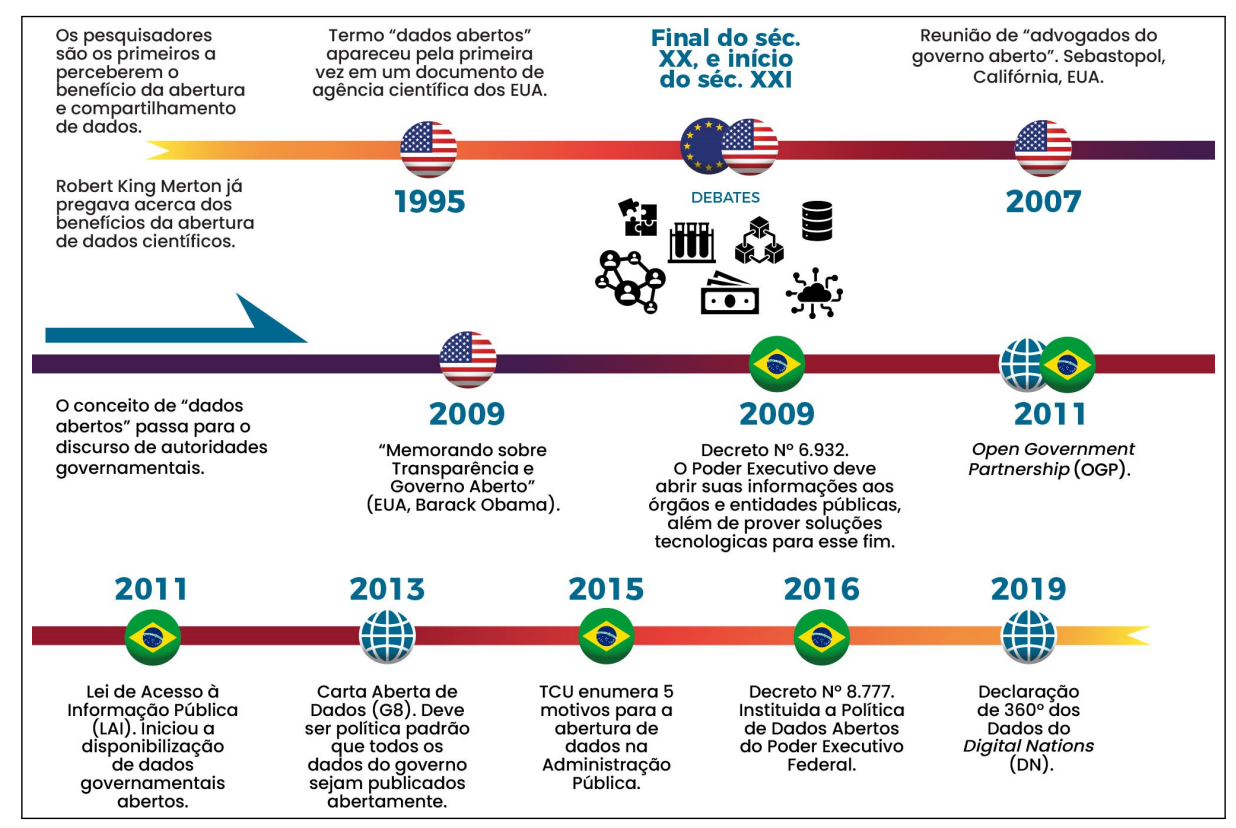

Figura 1. Cronologia com os principais marcos de abertura de dados. Fonte: Elaborado pelos autores (2021).

Mesmo já sendo objeto de interesse e discussão no meio científico, a temática Dados Abertos ganha força apenas no final do século XX, inicialmente em países com grande desenvolvimento tecnológico e engajamento de atores da sociedade. Ao final da primeira década do século XXI, os governos nacionais passam a adotar em seus discursos os benefícios da abertura de dados, incluindo o tema em suas agendas governamentais. Observa-se, no entanto, um grande salto no movimento a partir da segunda década do século XXI, com rápida expansão em alcance global entre as nações. As normatizações legais, a criação de políticas de abertura, a governança e os protocolos técnicos amadurecem a partir desse período, o que o caracteriza ainda como um fenômeno muito recente, passível, portanto, de muitos desafios.

Com relação aos desafios (Tabela 3) enfrentados pelo movimento, os resultados alcançados pela revisão de literatura também permitiram mapear e organizar as principais barreiras para a abertura de dados abertos governamentais em âmbito mundial.

\footnotetext{
${ }^{17}$ https://www.canada.ca/en/government/system/digital-government/digital-nations/digital-nations-data-360-declaration.html
} 


\begin{tabular}{|c|c|}
\hline Desafios & Descrição \\
\hline $\begin{array}{l}\text { Infraestrutura } \\
\text { dos dados }\end{array}$ & $\begin{array}{l}\text { A infraestrutura e o gerenciamento de dados governamentais se mostram } \\
\text { deficientes e inconsistentes devido a alterações frequentes nos datasets. }\end{array}$ \\
\hline $\begin{array}{l}\text { Acesso aos dados } \\
\text { e usabilidade }\end{array}$ & $\begin{array}{l}\text { A interface dos dados não possui um bom projeto de designer, afetando a } \\
\text { capacidade de localização, consumo e integração. Os portais geralmente } \\
\text { possuem uma abordagem técnica e orientada a desenvolvedores, não } \\
\text { contemplando os usuários finais e cidadãos comuns. }\end{array}$ \\
\hline Publicação & $\begin{array}{l}\text { Os datasets são geralmente incompletos, sem informações adicionais, com } \\
\text { falta de padrão e muitas vezes sem metadados significativos, evidenciando } \\
\text { problemas de interoperabilidade e em fontes de referência comuns. As } \\
\text { publicações de forma geral não utilizam todo o potencial da Web de Dados } \\
\text { e os preceitos do LOD, essenciais para conectar datasets heterogêneos e } \\
\text { descobrir recursos relacionados. }\end{array}$ \\
\hline $\begin{array}{l}\text { Políticos } \\
\text { e sociais }\end{array}$ & $\begin{array}{l}\text { Os dados de maior impacto social ainda permanecem amplamente fora } \\
\text { do escopo de abertura. A abertura de dados capacita os empoderados, } \\
\text { fortalecendo ainda mais aqueles que já têm poder e privando os demais. }\end{array}$ \\
\hline Econômicos & $\begin{array}{l}\text { Os dados abertos carecem de um modelo financeiro sustentável, além de } \\
\text { sofrerem críticas por facilitar a neoliberalização através da comercialização } \\
\text { dos serviços públicos. }\end{array}$ \\
\hline $\begin{array}{l}\text { Organização e } \\
\text { processos } \\
\text { internos }\end{array}$ & $\begin{array}{l}\text { Se as autoridades públicas não perceberem o valor para abrir dados, não } \\
\text { alocarão os recursos necessários para garantir a sua realização. Soma-se } \\
\text { a isso, a necessidade de proteger seus interesses pessoais como uma forte } \\
\text { razão para que mantenham alguns dados secretos ou inacessíveis. }\end{array}$ \\
\hline $\begin{array}{l}\text { Legais e de } \\
\text { privacidade }\end{array}$ & $\begin{array}{l}\text { A natureza dos dados abertos impõe novas demandas ao governo para } \\
\text { prover privacidade, segurança e impedir o uso indevido das informações. } \\
\text { Os governos devem determinar a política sobre quais dados devem ser } \\
\text { compartilhados e suas circunstâncias adequadas. }\end{array}$ \\
\hline $\begin{array}{l}\text { Capacitação } \\
\text { e treinamento }\end{array}$ & $\begin{array}{l}\text { Grande parte dos usuários não possui as habilidades necessárias para } \\
\text { avaliar a qualidade e a adequação ao uso de dados, além de não possuir o } \\
\text { alcance do que podem fazer com informações associadas. Soma-se a isso } \\
\text { a necessidade de estudantes recém-formados e profissionais que lidam com } \\
\text { tratamento e análise de dados serem devidamente treinados e capacitados } \\
\text { tecnicamente em várias competências interdisciplinares. }\end{array}$ \\
\hline
\end{tabular}

Tabela 3. Desafios para a abertura de dados governamentais. Fonte: Elaborado pelos autores (2021).

A Tabela 3 ajuda a compreender de forma sucinta quais as principais áreas em que os desafios se fazem presentes, apontando barreiras de natureza informacional, organizacional, política, legal e tecnológica. Percebe-se que mesmo com o desenvolvimento da área de tratamento e publicação dos dados, os governos ainda carecem de políticas públicas de dados mais bem definidas, com conjuntos de programas, ações e decisões tomadas pelos governos juntamente a entes públicos, privados e a própria sociedade, sendo esta última alijada do processo.

As quedas de representatividade de nações pioneiras, ricas e avançadas como EUA e Reino Unido também mostram que ser detentora de grande tecnologia não é sinônimo de qualidade para abertura, o que reforça ainda mais a importância a ser encarada nas políticas de dados e a seriedade na sua aplicação. O objetivo é assegurar o direito ao acesso aos dados de acordo com os preceitos e definições consolidados ao longo dos anos.

A análise de conteúdo realizada nas fontes documentais confirmou a hipótese formulada na presente pesquisa de que dados governamentais são publicados na rede por grande parte das nações de forma precária, tanto em aspectos tecnológicos (variados tipos de soluções) e licenças de uso (não aberto) para acesso e reúso de dados quanto nas práticas de organização da informação estabelecidas. Observou-se a utilização de vários tipos de soluções tecnológicas para publicação de dados, o que acarreta na produção de dados em diversos formatos e linguagens, gerando desafios às instituições de governo com interesse na publicização de seus dados em rede.

Em relação ao tratamento ou curadoria dos dados presentes nas bases dos governos pesquisados, percebem-se lacunas associadas a aspectos semânticos da informação, o que vai dificultar questões ligadas à interoperabilidade entre as comunidades de governo para prover condições necessárias à integração plena de informações heterogêneas entre sistemas e bases de dados disponíveis na Web (Bizer et al., 2009). Percebe-se a ausência de modelos ou padrões de organização e representação da informação usados para a constituição de suas bases de dados. Poderse-ia explorar o uso de regras de catalogação, padrões de metadados, vocabulários e ontologias (D. L. S. Lemos et al., 2020), vistos como instrumentos de organização seminais ao campo da CI que ajudam nos processos de classificação e indexação de dados na Web.

Tais práticas teóricas e metodológicas da CI junto às da CC viabilizam possíveis estratégias na busca de soluções 
inteligentes endereçadas à descrição de recursos de informação para a Web (D. L. Lemos e Souza, 2020; Machado et al., 2019). A valorização no uso de padrões para a descrição de recursos digitais em nível de estrutura, valor, conteúdo e comunicação de dados (Gilliland, 2016) ameniza problemas ligados à normalização, à qualidade e ao intercâmbio de descrições que, em certa medida, poderiam se valer das potencialidades oferecidas pelos princípios da Web Semântica e dos dados abertos ligados (Machado et al., 2019). De certa maneira, o que se pode considerar como dados de qualidade oriundos de um processo de curadoria digital são os descritos por esses princípios.

Nesse sentido, o emprego de sistemas de organização do conhecimento, a exemplo de taxonomias, tesauros, e até mesmo artefatos de representação mais sofisticados como ontologias, deveria ser considerado como pré-requisito nas ações de organização da informação, conforme advogam D. L. Lemos e Souza (2020), propiciando, assim, enriquecimento semântico aos metadados, organização mais significativa às bases do governo e, consequentemente, melhorias aos sistemas de recuperação da informação que buscam se alinhar com os preceitos cinco estrelas do padrão LOD. Logo, tal prática ajudaria a amenizar um dos maiores problemas para dados abertos governamentais na quinta estrela, qual seja o uso de vocabulários que permitam relacionar dados de diversas bases.

\section{CONSIDERAÇÕES FINAIS}

Se por um lado o movimento de abertura de dados governamentais experimenta um momento de consolidação em algumas iniciativas, por outro, desperta preocupação. O presente estudo constatou a perda de progresso por parte dos EUA e Reino Unido, especialmente pela falta de comprometimento político de suas estruturas administrativas com a qualidade dos dados disponibilizados seguindo definições trabalhadas ao longo dos anos pelos ativistas de dados.

Em contrapartida, é perceptível que algumas nações como Irlanda, França, Canadá e Coréia do Sul tomaram o protagonismo para o avanço do movimento. Identificou-se nesses países o claro respaldo governamental na construção das políticas públicas, com respeito à transparência, à disponibilização dos dados e boas práticas de governança.

A partir da conclusão do estudo tem-se o seguinte recorte com sugestões de aprimoramento: i) maior colaboração entre as organizações interessadas, focando no impacto das políticas para se ter resultados mais concretos; ii) criação de uma cultura de dados incorporada como padrão dentro das rotinas de trabalho; iii) mudança na mentalidade de abertura por "obrigação" para uma cultura em que a divulgação de dados seja incentivada, tornando claro além do aspecto legal, a sua importância social; iv) harmonização dos interesses políticos por trás da abertura de dados, frente à complexidade e os riscos que os servidores públicos enfrentam ao abrir os dados, como exposição de dados e privacidade.

Acrescenta-se a importância da promoção de eventos como os hackathons (Crusoe \& Ahlin, 2019), por agregar entusiastas a fim de explorar dados abertos, e assim propor projetos de software ou hardware fazendo uso desses dados. Brito, Costa, Garcia, e Meira (2014) vão além, condicionando maior participação cidadã a redução do papel do governo, reduzindo a este último apenas a entrega do dado bruto.

Países como Irlanda e Espanha já demonstraram ser possível incluir no desenvolvimento de suas ações a participação de iniciativas privadas de utilidade pública com origem na sociedade civil. Portanto, os resultados obtidos por essas nações devem ser observados mais atentamente a partir de agora, abrindo uma oportunidade para futuras pesquisas.

A partir da discussão, fica claro que apenas a formalização de uma política pública de dados não tem se mostrado suficiente. É necessário aplicá-la de forma responsável e criteriosa, com suporte de uma estrutura pública de governança comprometida com padrões de qualidade internacionais. Os procedimentos devem ser conduzidos de forma a garantir maior transparência e qualidade aos dados. Isso se aplica a toda a Administração Pública, perpassando gestores até servidores públicos.

O objetivo do artigo se cumpriu, logo, sua questão também respondida quando traz elementos sugestivos para o aumento dos investimentos em organização e representação da informação, governança e políticas de qualidade da Administração Pública orientada à gestão de dados.

Por fim, os desafios tecnológicos persistem, e as nações com maiores investimentos em governo digital conseguem disponibilizar uma infraestrutura mais robusta para lidar com a abertura de dados. A CI e CC também estão em sintonia e contribuindo para tornar a publicação e a representação dos dados o mais enriquecido possível, sendo o LOD uma iniciativa inovadora e sofisticada à disposição dos governos. Assim sendo, agrega-se à continuidade dessa pesquisa, uma sistematização de soluções e boas práticas na publicação de dados em LOD, especialmente pelo governo brasileiro, visando subsídios para o progresso de um governo aberto e interoperável na rede. 


\section{REFERÊNCIAS}

Afful-Dadzie, E., \& Afful-Dadzie, A. (2017). Open government data in africa: A preference elicitation analysis of media practitioners. Government Information Quarterly, 34(2), 244-255. doi: https://doi.org/10.1016/j.giq.2017.02.005

Altayar, M. S. (2018). Motivations for open data adoption: An institutional theory perspective. Government Information Quarterly, 35(4), 633-643. doi: https://doi.org/10.1016/j.giq.2018.09.006

Attard, J., Orlandi, F., Scerri, S., \& Auer, S. (2015). A systematic review of open government data initiatives. Government Information Quarterly, 32(4), 399-418. doi: https://doi.org/10.1016/j.giq.2015.07.006

Bardin, L. (2016). Análise de conteúdo. São Paulo: Edições 70.

Bauer, F., \& Kaltenböck, M. (2011). Linked open data: The essentials. Vienna: Edition Mono/Monochrom.

Berners-Lee, T. (2009). Putting government data online. Recuperado de https://www.w3.org/DesignIssues/GovData .html

Berners-Lee, T., Hendler, J., \& Lassila, O. (2001). The semantic web. Scientific american, 284 (5), 34-43.

Bizer, C., Health, T., \& Berners-Lee, T. (2009). Linked data-the story so far. International Journal on Semantic Web and Information Systems, 5, 1-12.

Blank, M. (2019). Open data maturity report 2019. European Data Portal. Recuperado de https://www.europeandataportal.eu/sites/default/files/ open_data_maturity_report_2019.pdf

Brito, K. S., Costa, M. A. S., Garcia, V. C., \& Meira, S. R. L. (2014). Brazilian government open data: implementation, challenges, and potential opportunities. In Proceedings of the 15th annual international conference on digital government research (p. 11-16).

Chatfield, A. T., \& Reddick, C. G. (2018). The role of policy entrepreneurs in open government data policy innovation diffusion: An analysis of australian federal and state governments. Government Information Quarterly, 35(1), 123-134. doi: https://doi.org/10.1016/j.giq.2017.10.004

Chignard, S. (2013). A brief history of open data (v. 29). Paris Tech Review. Recuperado de http://parisinnovationreview.com/articles-en/ a-brief-history-of-open-data

Crusoe, J. R., \& Ahlin, K. (2019). Users' activities for using open government data-a process framework. Transforming government: People, process and policy, 13(3/4), 213-236. doi: https://doi.org/10.1108/TG-04-2019-0028

Davies, T. (2010). Open data, democracy and public sector reform. Recuperado de http://www.opendataimpacts.net/ report/

Davies, T. G., \& Bawa, Z. A. (2012). The promises and perils of open government data (ogd). The Journal of Community Informatics, 8(2), 1-8. doi: https://doi.org/10.15353/joci.v8i2.3035

Gil, A. C. (2019). Como elaborar projetos de pesquisa. São Paulo: Atlas.

Gilliland, A. J. (2016). Setting the stage. In Introduction to metadata (3a. ed., p. 1-19). Los Angeles: Getty Research Institute. doi: http://dx.doi.org/10.2139/ssrn.2605828

González, J. A. M. (2011). Linguagens documentárias e vocabulários semênticos para a web: elementos conceituais. Bahia: EDUFBA.

Gray, J. (2014). Towards a genealogy of open data. In The paper was given at the general conference of the european consortium for political research in glasgow. doi: http://dx.doi.org/10.2139/ssrn.2605828

Huang, R., Wang, C., Zhang, X., Wu, D., \& Xie, Q. (2019). Design, develop and evaluate an open government data platform: a user-centred approach. The Electronic Library, 37(3), 550-562. doi: https://doi.org/10.1108/EL-02-20190037

International Federation of Library Associations and Institutions. (2009). Functional requirements for bibliographic records. study group on the functional requirements for bibliographic records. Recuperado de https://www.ifla.org/files/ assets/cataloguing/frbr/frbr_2008.pdf

Jetzek, T., Avital, M., \& Bjørn-Andersen, N. (2014). Generating sustainable value from open data in a sharing society. In International working conference on transfer and diffusion of it (p. 62-82). Berlin, Heidelberg: Springer.

Joudrey, D. N., Taylor, A. G., \& Miller, D. P. (2015). Introduction to cataloging and classification. Santa Barbara: ABC-CLIO.

Juana-Espinosa, S., \& Luján-Mora, S. (2019). Open government data portals in the european union: Considerations, development, and expectations. Technological Forecasting and Social Change, 149, 119769. doi: https://doi.org/10.1016/j.techfore.2019.119769

Kassen, M. (2018). Open data and its institutional ecosystems: A comparative cross-jurisdictional analysis of open data platforms. Canadian Public Administration, 61(1), 109-129. doi: https://doi.org/10.1111/capa.12251

Kučera, J., Chlapek, D., Klímek, J., \& Necaský, M. (2015). Methodologies and best practices for open data publication. In Dateso (p. 52-64). CEUR-WS.org.

Lancaster, F. W. (1986). Vocabulary control for information retrieval. Arlington: VA: Information Resources Press.

Lemos, D. L., \& Souza, R. R. (2020). Knowledge organization systems for the representation of multimedia resources on the web: A comparative analysis. Knowledge Organization, 47(4), 300-319. doi: https://doi.org/10.5771/09437444-2020-4-300

Lemos, D. L. S., Mendonça, F. M., \& Souza, R. R. (2020). Ontologias no suporte semântico na organização de acervos digitais em rede. In Representação do conhecimento, ontologias e linguagem: pesquisa aplicada em ciência da informação (p. 161-191). Curitiba: CRV.

Luna-Reyes, L. F., \& Najafabadi, M. M. (2019). The us open data initiative: The road ahead. Information Polity, 24 (2), 163-182. doi: https://doi.org/10.3233/IP-180106

Macedo, D. F., \& Lemos, D. L. S. (2019). Dados governamentais na rede linked open data: iniciativas nas esferas públicas brasileiras. In Encontro nacional de pesquisa em pós-graduação em ciência da informação (v. 20). Florianópolis.

Machado, L. M. O., Souza, R. R., \& Graça Simões, M. (2019). Semantic web or web of data? a diachronic study (1999 to 2017) of the publications of tim berners-lee and the world wide web consortium. Journal of the Association for Information Science and Technology, 70(7), 701-714. doi: https://doi.org/10.1002/asi.24111

Ministério do Planejamento. (2018). Plano de dados abertos 
vigência 12/2018 a 12/2020. Recuperado de https://plano .dados.planejamento.gov.br/

Open Government Partnership. (2011). Open government declaration. Recuperado de https:// www.opengovpartnership.org/process/joining-ogp/ open-government-declaration/

Organisation for Economic Co-operation and Development. (2019). Open, useful and re-usable data (ourdata) index: 2019. Paris: OECD Public Governance Policy Papers. Recuperado de http://www.oecd.org/gov/digital-government/ ourdata-index-policy-paper-2020.pdf

Pavlík, J., Hrnčírová, M., Stočes, M., Masner, J., \& Vaněk, J. (2020). Usability of iot and open data repositories for analyzing water pollution. a case study in the czech republic. ISPRS International Journal of Geo-Information, 9(10), 591. doi: https://doi.org/10.3390/ijgi9100591

Pinto, J. A., \& Almeida, M. B. (2020). Ontologias públicas sobre governo eletrônico. Brazilian Journal of Information Science: Research Trends, 14(3), e020003. doi: https://doi.org/10.36311/1940-1640.2020.v14n3.10105

Portal Brasileiro de Dados Abertos. (2020). Sobre o dados.gov.br. Recuperado de http://dados.gov.br/pagina/ sobre

Rautenberg, S., Souza, L., Dall'Agnol, J. M. H., \& Michelon, G. A. (2019). Guia prático para publicação de dados abertos conectados na web. Curitiba: Appris Editora.

Ribeiro, C. J. S., \& Almeida, R. F. (2011). Dados abertos governamentais (open government data): instrumento para exercício de cidadania pela sociedade. In Encontro nacional de pesquisa em pós-graduação em ciência da informação (v. 12). Brasília.

Saez Martin, A., Rosario, A. H. D., \& Pérez, M. D. C. C. (2016). An international analysis of the quality of open government data portals. Social science computer review, $34(3), 298-311$.

Santarem Segundo, J. E. (2015). Web semântica, dados ligados e dados abertos: uma visão dos desafios do brasil frente às iniciativas internacionais. In Encontro nacional de pesquisa em pós-graduação em ciência da informação (v. 16). João Pessoa.

Saxena, S. (2017). Open public data (opd) and the gulf cooperation council (gcc): challenges and prospects. Contemporary Arab Affairs, 10(2), 228-240. doi: https://doi.org/10.1080/17550912.2017.1297565

Schrock, A. R. (2016). Civic hacking as data activism and advocacy: A history from publicity to open government data. New media Es society, 18(4), 581-599. doi: https://doi.org/10.1177/1461444816629469

Shepherd, E., Bunn, J., Flinn, A., Lomas, E., Sexton, A., Brimble, S., ... Page, J. (2019). Open government data: critical information management perspectives. Records Management Journal, 29(1), 152-167. doi: https://doi.org/10.1108/RMJ-08-2018-0023

Silva, P. N., \& Pinheiro, M. M. K. (2015). Dados governamentais abertos e a lei de acesso à informação: diagnóstico nas universidades públicas federais brasileiras. In Encontro nacional de pesquisa em pós-graduação em ciência da informação (v. 16). João Pessoa.

Silva, P. N., \& Pinheiro, M. M. K. (2019). Execução da política de dados abertos no brasil: uma avaliação dos três anos do decreto $\mathrm{n}^{\circ} 8.777 / 2016$. Tendências da Pesquisa Brasileira em Ciência da Informação, 24(2). Recuperado de https://revistas.ancib.org/index.php/tpbci/article/view/ 495

Vetrò, A., Canova, L., Torchiano, M., Minotas, C. O., Iemma, R., \& Morando, F. (2016). Open data quality measurement framework: Definition and application to open government data. Government Information Quarterly, 33(2), 325-337. doi: https://doi.org/10.1016/j.giq.2016.02.001

Victorino, M. C., Shiessl, M., Oliveira, E. C., Ishikawa, E., de Holanda, M. T., \& de Lima Hokama, M. (2017). Uma proposta de ecossistema de big data para a análise de dados abertos governamentais concetados. Informação $\mathcal{E}$ sociedade, 27(1). Recuperado de https://periodicos.ufpb.br/ ojs2/index.php/ies/article/view/29299

Wang, D., Chen, C., \& Richards, D. (2018). A prioritizationbased analysis of local open government data portals: A case study of chinese province-level governments. Government Information Quarterly, 35(4), 644-656. doi: https://doi.org/10.1016/j.giq.2018.10.006

Wang, V., \& Shepherd, D. (2020). Exploring the extent of openness of open government data-a critique of open government datasets in the uk. Government Information Quarterly, 37(1), 101405. doi: https://doi.org/10.1016/j.giq.2019.101405

Wang, V., Shepherd, D., \& Button, M. (2019). The barriers to the opening of government data in the uk: A view from the bottom. Information Polity, 24(1), 59-74. doi: https://doi.org/10.3233/IP-180107

Wirtz, B. W., Piehler, R., Thomas, M.-J., \& Daiser, P. (2016). Resistance of public personnel to open government: A cognitive theory view of implementation barriers towards open government data. Public Management Review, 18(9), 1335-1364. doi: https://doi.org/10.1080/14719037.2015.1103889

Yi, M. (2019). Exploring the quality of government open data. The Electronic Library, 37(1), 35-48. doi: https://doi.org/10.1108/EL-06-2018-0124

Young, A., \& Verhulst, S. (2016). The global impact of open data: Key findings from detailed case studies around the world (S.1 ed.). O'Reilly.

Como citar este artigo (APA):

Macedo, D. F. \& Lemos, D. L. S. (2021). Dados abertos governamentais: iniciativas e desafios na abertura de dados no Brasil e outras esferas internacionais. AtoZ: novas práticas em informação e conhecimento, 10(2), 14 - 26. Recuperado de: http://dx.doi.org/10.5380/ atoz.v10i2.77737 